\title{
Design of Robust Pulses to Insufficient Synchronization for OFDM/OQAM Systems in Doubly Dispersive Channels
}

\author{
Yu Zhao, ${ }^{1}$ Xihong Chen, ${ }^{1}$ Lunsheng Xue, ${ }^{1}$ and Qun Zhang ${ }^{2}$ \\ ${ }^{1}$ Air and Missile Defense College, Air Force Engineering University, Xian 710051, China \\ ${ }^{2}$ Information and Navigation College, Air Force Engineering University, Xi'an 710077, China \\ Correspondence should be addressed to Yu Zhao; sunzy54321@163.com
}

Received 10 January 2015; Revised 3 May 2015; Accepted 4 May 2015

Academic Editor: Oleg V. Gendelman

Copyright (c) $2015 \mathrm{Yu}$ Zhao et al. This is an open access article distributed under the Creative Commons Attribution License, which permits unrestricted use, distribution, and reproduction in any medium, provided the original work is properly cited.

\begin{abstract}
This paper presents a pulse shaping method robust to insufficient synchronization in orthogonal frequency division multiplexing with offset quadrature amplitude modulation (OFDM/OQAM) systems over doubly dispersive (DD) channels. The proposed pulse is designed as a linear combination of several well localized Hermite functions. The coefficients optimization problem is modeled as a nonconvex constrained fractional programming problem based on the signal-to-interference ratio (SIR) maximization criterion. An efficient iterative algorithm is applied to simplify the problem to a series of quadratically constrained quadratic program (QCQP) problems which can be solved by semidefinite relaxation (SDR) method. Simulation results show that the proposed pulse is superior to traditional pulses with respect to SIR performance over DD channels in the presence of carrier frequency offset (CFO) and timing offset (TO).
\end{abstract}

\section{Introduction}

Multicarrier (MC) transmission is employed in various communication systems due to its robustness against time dispersion caused by multipath propagation. The systems are generally lattice structures formed by time-frequency shifts of a prototype pulse. Orthogonal frequency division multiplexing (OFDM) is a conventional MC technology for some advantages of efficient implementation through FFT technique and a simple equalization in frequency domain. However, in OFDM systems, the cyclic prefix (CP) used to prevent intersymbol interference (ISI) will cost a loss of spectral efficiency and increase the power consumption. Moreover, the rectangular prototype pulse which exhibits a sinc-shaped frequency response will result in serious intercarrier interference (ICI) in frequency dispersion channels.

In order to counteract the drawbacks of OFDM systems, some pulse-shaping OFDM (PS-OFDM) systems have been proposed [1-4]. These systems are more robust to frequency dispersion caused by Doppler effect and carrier frequency offset (CFO) by employing prototype pulses with lower sidelobe and faster spectral decay. However, the underlying requirement in PS-OFDM systems is that the prototype pulses must be orthogonal or biorthogonal to each other. From frame theory, a well localized orthogonal pulses set cannot be achieved for unit density structures due to BalianLow theorem. In the literature, many lattice structures employ half lattice density which results in a loss of bandwidth efficiency.

Recently, Filter Bank Multicarrier (FBMC) technology has been intensively studied as an alternative to OFDM in order to improve the robustness against various dispersive channels and to increase the bandwidth efficiency [5]. Among all the FBMC systems, the most popular one is the OFDM/OQAM which has already been introduced in the TIA's Digital Radio Technical Standards and considered in WRAN (IEEE802.22) [6]. Its principle is to introduce a half symbol duration time offset between the real and imaginary parts of a QAM constellation and transmit them separately on each subcarrier. With this offset QAM approach, the orthogonality constraint only holds in real field. Therefore, a well localized pulse can be introduced in OFDM/OQAM systems with unit lattice density. 
The time and frequency dispersion of the DD channel is susceptible to cause ISI and ICI in multicarrier transmission. In addition, OFDM/OQAM systems suffer from high sensitivity to some desynchronization like CFO and TO $[7,8]$. $\mathrm{CFO}$ is mainly caused by the different local oscillators of the transmitter and receiver. TO may occur when the actual sampling instant at the receiver is different from the optimal one. Normally, CFO and TO estimation is carried out prior to the demodulation. However, the estimation is usually not perfect. Therefore, design of robust pulses to insufficient synchronization over DD channels is an attractive research topic. In [9], the WSSUS pulse shape optimization is translated to a convex constraint quasi-convex maximization problem. In [10], a pulse shape robust to Doppler effect is constructed with optimized combination of Hermite functions. In [11], a novel cost function for optimization of the filter bank prototype pulse is proposed to achieve robust performance over DD channels. In [12], an OFDM/QAM pulse that is optimal with respect to interference due to $\mathrm{CFO}$ is introduced.

In this paper, a robust prototype pulse modeled as the linear combination of normalized Hermite functions is proposed for OFDM/OQAM systems. The proposed pulse is designed to be robust to channel dispersion and insufficient synchronization. Firstly, the exact SIR expression of the demodulated symbol versus CFO and TO is derived in the assumption of wide sense stationary uncorrelated scattering (WSSUS) channel. Then, the coefficients of Hermite functions are optimized based on SIR maximization criterion. Finally, the optimization problem is simplified to a series of QCQP problems by using an effective iterative algorithm. By taking into account the semidefinite relaxation, the QCQP problems can be translated to semidefinite programming (SDP) problems which are easy to be solved. Theoretical analysis and simulation results show that the proposed pulse outperforms traditional pulses especially in the case of seriously dispersive channels with large CFO and TO.

The rest of this paper is organized as follows. The system model of OFDM/OQAM is described in the next section. In Section 3, design of the robust pulse is formulated as a SIR maximization problem. In Section 4, the proposed optimization algorithm is presented and performance of the optimal pulse is tested by numerical simulations in Section 5 . Finally, some conclusions are drawn in Section 6.

\section{System Model}

Instead of using complex symbols in OFDM/QAM systems, the real-valued symbols modulated by offset QAM are transmitted on each subcarrier in OFDM/OQAM scheme. With the synthesis basis functions obtained by the time-frequency translated version of the prototype pulse, the transmitted baseband signal can be written in the following analytic form [6]:

$$
s(t)=\sum_{k \in \mathbb{Z}} \sum_{m=0}^{M-1} a_{m, k} \underbrace{e^{j \phi_{m k}} e^{j 2 \pi m F_{0} t} g\left(t-k \tau_{0}\right)}_{g_{m, k}(t)},
$$

where $\phi_{m k}=(\pi / 2)(m+k)$ is the phase term related to the staggering rule of OQAM, $F_{0}$ is the intercarrier spacing, $\tau_{0}$ is the symbol duration time $\left(F_{0} \tau_{0}=1 / 2\right), M$ is the subcarrier number which is supposed to be even, and $a_{m, k}$ denotes the real-valued symbol located on the $m$ th subcarrier at time instant $k \tau_{0}$. The symbols are obtained by introducing half a symbol spacing between the real and imaginary parts of the QAM complex-valued symbols. They are assumed to be independent and identically distributed with zero mean and average energy $\sigma_{a}^{2} \cdot g(t)$ denotes the real-valued even prototype pulse in this paper.

Assuming a distortion-free channel, the following orthogonality condition is required for complete reconstruction of the data symbols:

$$
\begin{aligned}
& \left\langle g_{m, k}(t), g_{n, l}(t)\right\rangle_{R} \\
& =\mathfrak{R}\left\{(j)^{k-l+m-n+(k-l)(n+m)} A_{g}\left((n-m) \tau_{0},(l-k) F_{0}\right)\right\} \\
& =\delta(m-n, k-l)
\end{aligned}
$$

where $\mathfrak{R}\{\cdot\}$ is the real part operator and $\delta(\cdot, \cdot)$ denotes the 2D Kronecker delta function. And $A_{g}(\tau, v)$, the ambiguity function of $g(t)$, is defined by

$$
A_{g}(\tau, v)=\int_{R} g\left(t+\frac{\tau}{2}\right) g^{*}\left(t-\frac{\tau}{2}\right) e^{-j 2 \pi v t} d t
$$

It is observed that $A_{g}(\tau, v)$ is real-valued as long as $g(t)$ is an even function [6]. Hence, the orthogonality can be ensured by finding a real and even prototype pulse of which the ambiguity function satisfies

$$
A_{g}\left(2 u \tau_{0}, 2 v F_{0}\right)=\delta(u, v), \quad(u, v) \in \mathbb{Z} .
$$

The baseband doubly dispersive channel can be modeled as a random time-varying system $H$ with impulse response $h(t, \tau)$ [13]. Therefore, considering the noiseless case, the received signal can be expressed as

$$
\begin{aligned}
r(t) & =H[s(t)] \\
& =\int_{0}^{\tau_{\max }} \int_{-f_{d}}^{f_{d}} H(\tau, v) s(t-\tau) e^{j 2 \pi v t} d \tau d v,
\end{aligned}
$$

where $\tau_{\max }$ and $f_{d}$ are the maximum multipath delay spread and Doppler frequency, respectively. We denote $\theta=\tau_{\max } \times f_{d}$ as the channel spread factor. In general, practical wireless channels are usually underspread and satisfy $\theta \ll 1$. The delay-Doppler spread function $H(\tau, v)$ is the Fourier transform of $h(t, \tau)$ with respect to $t$. In the WSSUS assumption, the channel is characterized by the second-order statistics of $H(\tau, v)$, that is, the scattering function $S_{H}(\tau, v)$ :

$$
\begin{aligned}
& E\left[H(\tau, v) H^{*}\left(\tau^{\prime} v^{\prime}\right)\right] \\
& \quad=S_{H}(\tau, v) \delta\left(\tau-\tau^{\prime}\right) \delta\left(v-v^{\prime}\right),
\end{aligned}
$$

where $E[\cdot]$ denotes the expectation operator. Moreover, it is assumed that $E[H(\tau, v)]=0$. In this paper, we set $\int_{\tau} \int_{v} S_{H}(\tau, v) d \tau d v=1$, which means that the channel has no overall path loss. 


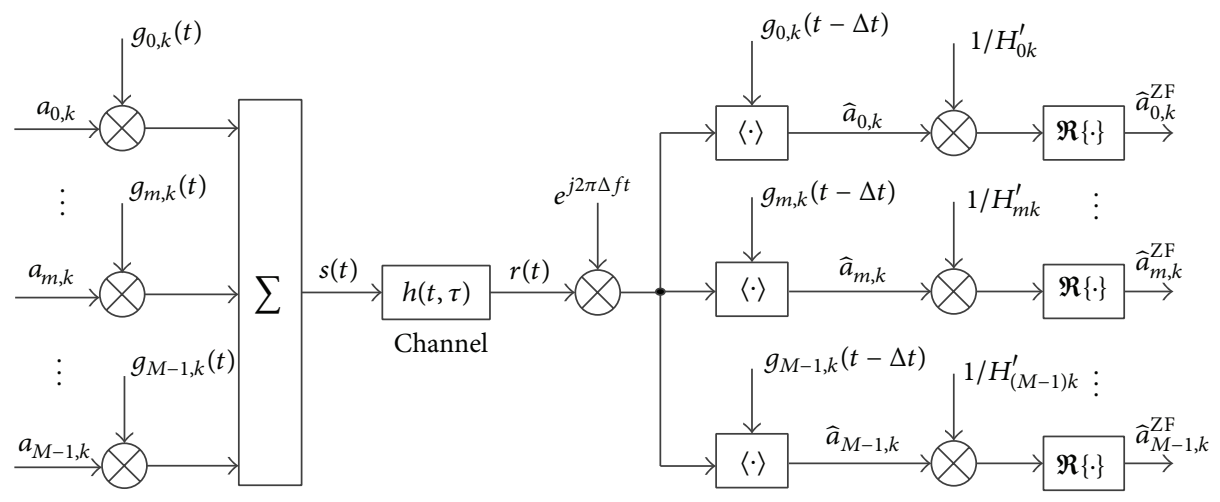

FIgURE 1: Baseband equivalent of an OFDM/OQAM system with $M$ subcarriers in the presence of CFO and TO.

At the receiver, the demodulated signal $\widehat{a}_{m_{0}, k_{0}}$ for timefrequency position $\left(m_{0}, k_{0}\right)$ is performed by a projection of the received signal $r(t)$ onto the set of functions $\left\{g_{m_{0}, k_{0}}(t)\right\}$ with the modified inner product. Consider

$$
\begin{aligned}
\widehat{a}_{m_{0}, k_{0}} & =\left\langle r(t), g_{m_{0}, k_{0}}(t)\right\rangle_{R} \\
& =\mathfrak{R}\left\{\int_{R} r(t) g_{m_{0}, k_{0}}^{*}(t) d t\right\} \\
& =\sum_{k \in \mathbb{Z}} \sum_{m=0}^{M-1} a_{m, k}\left\langle H\left(g_{m, k}(t)\right), g_{m_{0}, k_{0}}(t)\right\rangle_{R} .
\end{aligned}
$$

\section{Pulse Design and SIR Criterion}

3.1. Effects of TO, CFO, and DD Channels. Considering the insufficient synchronization, we introduce a timing offset $\Delta t$ and a carrier frequency offset $\Delta f$. Then, the baseband equivalent of an OFDM/OQAM system with $M$ subcarriers in the presence of CFO and TO is depicted in Figure 1. In general, the demodulated data symbol before the real part extraction can be expressed as

$$
\begin{aligned}
& \widehat{a}_{m_{0}, k_{0}} \\
& \quad=\left\langle e^{j 2 \pi \Delta f t} r(t), g_{m_{0}, k_{0}}(t-\Delta t)\right\rangle \\
& \quad=\sum_{k \in \mathbb{Z}} \sum_{m=0}^{M-1} a_{m, k}\left\langle e^{j 2 \pi \Delta f t} H\left(g_{m, k}(t)\right), g_{m_{0}, k_{0}}(t-\Delta t)\right\rangle \\
& \quad=\sum_{k \in \mathbb{Z}} \sum_{m=0}^{M-1} a_{m, k} \Theta_{m_{0}, k_{0} ; m, k}^{\Delta t, \Delta f}
\end{aligned}
$$

where $\Theta_{m_{0}, k_{0} ; m, k}^{\Delta t, \Delta f}$ is given in (A.1) (see Appendix A). If we set $m=m_{0}$ and $k=k_{0}$, then the desired section of the data symbol can be expressed as

$$
\begin{aligned}
& a_{m_{0}, k_{0}} \Theta_{m_{0}, k_{0} ; m_{0}, k_{0}}^{\Delta t, \Delta f} \\
& \quad=a_{m_{0}, k_{0}} e^{j 2 \pi\left[\Delta f\left(\Delta t+k_{0} \tau_{0}\right)+m_{0} F_{0} \Delta t\right]} \int_{\tau} \int_{v} e^{j 2 \pi\left[v\left(\Delta t+k_{0} \tau_{0}\right)-m_{0} F_{0} \tau\right]} H(\tau, \\
& \quad v) A_{g}^{*}(\tau-\Delta t, v+\Delta f) d \tau d v \\
& =e^{j 2 \pi\left[\Delta f\left(\Delta t+k_{0} \tau_{0}\right)+m_{0} F_{0} \Delta t\right]} \Lambda_{H}\left(\tau_{\max }, f_{d}, \Delta t, \Delta f\right) a_{m_{0}, k_{0}} .
\end{aligned}
$$

Equation (9) shows that the desired section of the data symbol at the receiver is rotated by a phasor $2 \pi[\Delta f(\Delta t+$ $\left.\left.k_{0} \tau_{0}\right)+m_{0} F_{0} \Delta t\right]$, which is caused by the CFO and TO. To compensate the phase rotation, the demodulated data symbol $\widehat{a}_{m_{0}, k_{0}}$ is equalized with a single complex-tap. We can assume the equalizer to be a simple one-tap zero-forcing (ZF) equalizer at each subcarrier and each time instant, which eventually leads the equalized data symbol after real-parttaken operation to

$$
\widehat{a}_{m_{0}, k_{0}}^{\mathrm{ZF}}=\sum_{k \in \mathbb{Z}} \sum_{m=0}^{M-1} a_{m, k} \mathfrak{R}\left\{\frac{\Theta_{m_{0}, k_{0} ; m, k}^{\Delta t, \Delta f}}{H_{m_{0} k_{0}}^{\prime}}\right\},
$$

where $H_{m_{0} k_{0}}^{\prime}=e^{j 2 \pi\left[\Delta f\left(\Delta t+k_{0} \tau_{0}\right)+m_{0} F_{0} \Delta t\right]}$ is the ZF equalizer coefficient at the $\left(m_{0}, k_{0}\right)$ subcarrier. It is then easy to separate the equalized data symbol into the useful part and ICI/ISI part; that is,

$$
\begin{aligned}
\widehat{a}_{m_{0}, k_{0}}^{\mathrm{ZF}}= & a_{m_{0} k_{0}} \mathfrak{R}\left\{\frac{\Theta_{m_{0}, k_{0} ; m_{0}, k_{0}}^{\Delta t, \Delta f}}{H_{m_{0} k_{0}}^{\prime}}\right\} \\
& +\sum_{k \neq k_{0}} \sum_{m \neq m_{0}} a_{m, k} \mathfrak{R}\left\{\frac{\Theta_{m_{0}, k_{0} ; m, k}^{\Delta t, \Delta f}}{H_{m_{0} k_{0}}^{\prime}}\right\} \\
= & \Re\left\{\Lambda_{H}\left(\tau_{\max }, f_{d}, \Delta t, \Delta f\right)\right\} a_{m_{0} k_{0}}+J_{m_{0} k_{0}} .
\end{aligned}
$$

Concerning the useful portion, the desired symbol is attenuated by $\Lambda_{H}\left(\tau_{\max }, f_{d}, \Delta t, \Delta f\right)$ which is caused by the DD channel, TO, and CFO. 
By taking into account the statistically independent property of the data symbols $a_{m, k}$ and the WSSUS assumption, the total energy of the equalized symbol can be calculated as (see Appendix A)

$$
E_{r}\left(\tau_{\max }, f_{d}, \Delta t, \Delta f\right)=\frac{1}{2} \sigma_{a}^{2} \sum_{k \in \mathbb{Z}} \sum_{m=0}^{M-1} \int_{\tau} \int_{v} S_{H}(\tau, v)\left|A_{g}\left(\left(k-k_{0}\right) \tau_{0}+\tau-\Delta t,\left(m-m_{0}\right) F_{0}+v+\Delta f\right)\right|^{2} d \tau d v
$$

Then, the desired signal energy can be calculated as

$$
\begin{aligned}
E_{S} & \left(\tau_{\max }, f_{d}, \Delta t, \Delta f\right) \\
& =\frac{1}{2} \sigma_{a}^{2} \int_{\tau} \int_{v} S_{H}(\tau, v)\left|A_{g}(\tau-\Delta t, v+\Delta f)\right|^{2} d \tau d v .
\end{aligned}
$$

The interference energy can be calculated as

$$
E_{I}\left(\tau_{\max }, f_{d}, \Delta t, \Delta f\right)=\frac{1}{2} \sigma_{a}^{2} \sum_{k \neq k_{0}} \sum_{m \neq m_{0}} \int_{\tau} \int_{v} S_{H}(\tau, v)\left|A_{g}\left(\left(k-k_{0}\right) \tau_{0}+\tau-\Delta t,\left(m-m_{0}\right) F_{0}+v+\Delta f\right)\right|^{2} d \tau d v
$$

Hence, the SIR expression is defined by

$$
\operatorname{SIR}\left(\tau_{\max }, f_{d}, \Delta t, \Delta f\right)=\frac{E_{S}}{E_{I}}
$$

Equations (13)-(15) illustrate that the SIR depends on the prototype pulse through its ambiguity function when the channel scattering function, CFO, and TO are confirmed. Various prototype pulses, such as square-root raised-cosine (SRRC) [14] and isotropic orthogonal transform algorithm (IOTA) [15] pulses and Hermite pulse (HP) design based on the Hermite functions [16], have been studied. With SRRC pulse, one can get good frequency attenuation property, while IOTA and HP pulses lead to good and identical time and frequency localization. Additionally, the authors in [17] realized a Nyquist pulse in both time and frequency, which is referred to as optimal finite duration pulse (OFDP), by minimizing the out-of-band energy of a time-limited pulse. However, the above pulses are highly sensitive to desynchronization over DD channels. Therefore, in this paper, we search for the optimal prototype pulse based on SIR maximization criterion:

$$
g_{\text {opt }}(t)=\arg \max _{g(t)} \operatorname{SIR}
$$

3.2. SIR Maximization Criterion Based on the Optimal Pulse. The optimal pulse is researched as a linear combination of several Hermite functions which are maximally localized in both time and frequency [18]. The $n$th order Hermite function $h_{n}(t)$ is given by

$$
h_{n}(t)=\left(\frac{2^{1 / 4}}{\sqrt{2^{n} n !}}\right) H_{n}(\sqrt{2 \pi t}) e^{-\pi t^{2}}
$$

where $H_{n}(t)$ is the $n$th order Hermite polynomial which can be computed recursively by $H_{0}(t)=1, H_{1}(t)=2 t$, and $H_{n+1}(t)=2 t H_{n}(t)-2 n H_{n-1}(t)$. We note that the Hermite function corresponding to $n=0$ is the Gaussian pulse and provides the best time and frequency localization. It has been proved that $h_{n}(t)$ is the eigenvector of its Fourier transformation: $F\left\{h_{n}(t)\right\}=\lambda_{n} h_{n}(t)$. Moreover, $\lambda_{n}=e^{-j n \pi / 2}$ is the corresponding eigenvalue. Therefore, if we choose $n=2 l$, $l=0,1,2, \ldots$, both $h_{n}(t)$ and its Fourier transformation are real and even functions. Finally, the proposed optimal pulse can be expressed as

$$
g(t)=\sum_{l=0}^{L-1} \alpha_{l} h_{2 l}(t) .
$$

Equation (18) indicates that the $L$ most concentrated Hermite functions are used. The coefficients $\alpha_{l}$ are assumed real. Substituting (18) into (3), we obtain

$$
A_{g}(\tau, v)=\sum_{l=0}^{L-1} \sum_{q=0}^{L-1} \alpha_{l} \alpha_{q} A_{l q}(\tau, v),
$$

where $A_{l q}(\tau, v)=\int_{R} h_{2 l}(t+\tau / 2) h_{2 q}{ }^{*}(t-\tau / 2) e^{-j 2 \pi v t} d t$ and they can be calculated according to [19]. Using (13), (14), (15), and (19), after a few steps of straightforward manipulations, we obtain

$$
\operatorname{SIR}=\frac{\sum_{0 \leq l, q, l^{\prime}, q^{\prime} \leq L-1} \alpha_{l} \alpha_{q} \alpha_{l^{\prime}} \alpha_{q^{\prime}} S\left(l, q, l^{\prime}, q^{\prime}\right)}{\sum_{0 \leq l, q, l^{\prime}, q^{\prime} \leq L-1} \alpha_{l} \alpha_{q} \alpha_{l^{\prime}} \alpha_{q^{\prime}} I\left(l, q, l^{\prime}, q^{\prime}\right)}
$$

with

$$
\begin{aligned}
& S\left(l, q, l^{\prime}, q^{\prime}\right)=\int_{\tau} \int_{v} S_{H}(\tau, v) A_{l q}(\tau-\Delta t, v+\Delta f) \\
& \quad \cdot A_{l^{\prime} q^{\prime}}^{*}(\tau-\Delta t, v+\Delta f) d \tau d v, \\
& I\left(l, q, l^{\prime}, q^{\prime}\right)=\int_{\tau} \int_{v} S_{H}(\tau, v) \\
& \quad \cdot \sum_{k \neq k_{0}} \sum_{m \neq m_{0}} A_{l q}\left(\left(k-k_{0}\right) \tau_{0}+\tau-\Delta t,\left(m-m_{0}\right) F_{0}+v+\Delta f\right) \\
& \cdot A_{l^{\prime} q^{\prime}}^{*}\left(\left(k-k_{0}\right) \tau_{0}+\tau-\Delta t,\left(m-m_{0}\right) F_{0}+v+\Delta f\right) d \tau d v .
\end{aligned}
$$


To precisely evaluate the SIR, it is sufficient to consider only the energy perturbation from neighboring symbols with negligible performance loss by employing the well localized pulses. Therefore, we consider $\left|k-k_{0}\right| \leq 4$ and $\mid m-$ $m_{0} \mid \leq 4$ in (22). Finally, the optimization task amounts to finding the coefficients $\alpha_{l}$ so that the objective function $\operatorname{SIR}\left(\tau_{\max }, f_{d}, \Delta t, \Delta f\right)$ is maximized.

\section{Optimization Strategy and Algorithm}

4.1. Optimization Problem Formulation. The optimization of the prototype pulse is achieved by the optimization of the coefficients $\alpha_{l}$ that maximize the SIR expression in (20). In order to get simplified, easier-to-manipulate expressions, let a denote the $L \times 1$ column vector containing the coefficients to be optimized: $\mathbf{a}=\left[\begin{array}{llll}\alpha_{0} & \alpha_{2} & \cdots & \alpha_{L-1}\end{array}\right]^{T}$. Then, we introduce the $L^{2} \times 1$ column vector $\mathbf{w}=\mathbf{a} \otimes \mathbf{a}$, where $\otimes$ denotes the matrix Kronecker product. As a function of $\mathbf{w}$, the SIR expression in (20) can be reorganized in the following matrix form:

$$
\operatorname{SIR}=\frac{\mathbf{w}^{T} \mathbf{S w}}{\mathbf{w}^{T} \mathbf{I w}},
$$

where $\mathbf{S}=\left(s_{i j}\right)$ and $\mathbf{I}=\left(i n_{i j}\right)$ and $0 \leq i, j \leq L^{2}-1$ are symmetric and positive definite (SPD) matrices, respectively, defined by $s_{i j}=S\left(l, q, l^{\prime}, q^{\prime}\right)$ and $i n_{i j}=I\left(l, q, l^{\prime}, q^{\prime}\right)$, where $i=l L+q$ and $j=l^{\prime} L+q^{\prime}$. The ambiguity function $A_{g}(\tau, v)$ can also be reorganized in the following matrix form:

$$
A_{g}(\tau, v)=\mathbf{a}^{T} \mathbf{A}(\tau, v) \mathbf{a},
$$

where $\mathbf{A}(\tau, v)=\left(a_{i j}\right)$ and $0 \leq i, j \leq L-1$ is defined by $a_{i j}=$ $A_{i j}(\tau, v)$. In [16], it has been proposed that the orthogonality constraints (4) were applied at the origin position $(u, v)=0$ and other $L-1$ nearest grid points around it. The operation relies on the good localization property of $g(t)$. Finally, substituting (24) in (4), the coefficients a can be determined by solving the resulting $L$ equations.

Since the ambiguity function is real-valued, the orthogonality conditions in (4) can be transformed to

$$
\left|A_{g}\left(2 u \tau_{0}, 2 v F_{0}\right)\right|^{2}=\delta(u, v) .
$$

Substituting (19) into (25), we obtain

$$
\mathbf{w}^{T} \mathbf{B}(u, v) \mathbf{w}=\delta(u, v),
$$

where $\mathbf{B}(u, v)=\left(b_{i j}\right)$ and $0 \leq i, j \leq L^{2}-1$, and it is defined by $b_{i j}=A_{l q}\left(2 u \tau_{0}, 2 v F_{0}\right) A_{l^{\prime} q^{\prime}}\left(2 u \tau_{0}, 2 v F_{0}\right)$, where $i=l L+q$ and $j=l^{\prime} L+q^{\prime}$.

Considering the effect of time and frequency dispersion in DD channels, the constraints on the nulls of the ambiguity function are not necessary [11]. Therefore, we simply relax the constraint (26) to $\mathbf{w}^{T} \mathbf{B}(0,0) \mathbf{w}=1$. Besides the orthogonality constraints on $\mathbf{w}$, there are some other inherent constraints due to the operation of matrix Kronecker product. After some computations, reported in Appendix B, we find that two kinds of equality constraints and one inequality constraint are introduced: $\mathbf{C w}=0, \mathbf{w}^{T} \mathbf{D}_{i j} \mathbf{w}=0$, and $\mathbf{G w} \geq 0$. Eventually, the optimization problem can be formulated as follows:

$$
\begin{aligned}
(\mathrm{P}): \max _{\mathbf{w}} & \frac{\mathbf{w}^{T} \mathbf{S w}}{\mathbf{w}^{T} \mathbf{I} \mathbf{w}} \\
\text { s.t. } & C 1: \mathbf{w}^{T} \mathbf{B}(0,0) \mathbf{w}=1 \\
& C 2: \mathbf{w}^{T} \mathbf{D}_{i j} \mathbf{w}=0, \quad 0 \leq i, j \leq L^{2}-1 \\
& C 3: \mathbf{C w}=0 \\
& C 4: \mathbf{G w} \geq 0 .
\end{aligned}
$$

4.2. Iterative Algorithm for SIR Maximization. As the optimization problem $(\mathrm{P})$ is a fractional programming problem, the objective function (OF) is nonconvex in this case. This kind of problem is NP-hard generally. In order to solve the problem, it is convenient to adopt Dinkelbach's parametric approach [20]. By defining $\Omega$ as the set of feasible solutions of problem $(\mathrm{P})$, the equivalent parametric problem associated with this problem can be expressed as

$$
\begin{aligned}
& F(\lambda)=\max _{\mathbf{w} \in \Omega}\left[f(\lambda, \mathbf{w})=\mathbf{w}^{T} \mathbf{S} \mathbf{w}-\lambda \mathbf{w}^{T} \mathbf{I} \mathbf{w}\right], \\
& \mathbf{w}(\lambda)=\arg \max _{\mathbf{w} \in \Omega} f(\lambda, \mathbf{w}) .
\end{aligned}
$$

If there exists $\lambda^{*} \geq 0$ for which $F\left(\lambda^{*}\right)=0$, it has been proved that $\mathbf{w}^{*}=\mathbf{w}\left(\lambda^{*}\right)$ is the optimal solution of problem (P) [20]. Then, an effective iterative algorithm which is known as Dinkelbach's method is proposed in Algorithm 1.

By employing the above iterative algorithm, problem (P) can be transformed to a series of QCQP problems as follows:

$$
\begin{aligned}
& \max _{\mathbf{w}} \mathbf{w}^{T}\left(\mathbf{S}-\lambda_{i-1} \mathbf{I}\right) \mathbf{w}, \\
& \text { s.t. } C 1, C 2, C 3, C 4 .
\end{aligned}
$$

However, considering the variety of $\lambda$, the optimization problem in (29) is a nonconvex quadratic program problem. Fortunately, the semidefinite relaxation (SDR) method can be applied to generate the suboptimal solutions with quite encouraging results [21]. It is crucial for the SDR method to observe that $\mathbf{w}^{T} \mathbf{H w}=\operatorname{Tr}\left(\mathbf{H}\left(\mathbf{w} \mathbf{w}^{T}\right)\right)$, where $\operatorname{Tr}(\cdot)$ stands for the trace of a matrix. To get simplified expressions, we introduce a matrix variable $\mathbf{M}=\mathbf{w} \mathbf{w}^{T}$, which is equivalent to $\mathbf{M}$ being a rank-one and positive semidefinite (PSD) matrix. Therefore, we can obtain the following equivalent formulation of (29):

$$
\begin{array}{ll}
\max _{\mathbf{M}} & \operatorname{Tr}\left(\left(\mathbf{S}-\lambda_{i-1} \mathbf{I}\right) \mathbf{M}\right) \\
\text { s.t. } & C 1: \operatorname{Tr}(\mathbf{B}(0,0) \mathbf{M})=1 \\
& C 2: \operatorname{Tr}\left(\mathbf{D}_{i j} \mathbf{M}\right)=0, \quad 0 \leq i, j \leq L^{2}-1 \\
& C 3: \operatorname{Tr}\left(\mathbf{C}^{T} C \mathbf{M}\right)=0 \\
& C 4: \mathbf{G w} \geq 0 \\
& C 5: \mathbf{M} \geq 0, \quad \operatorname{rank}(\mathbf{M})=1 .
\end{array}
$$




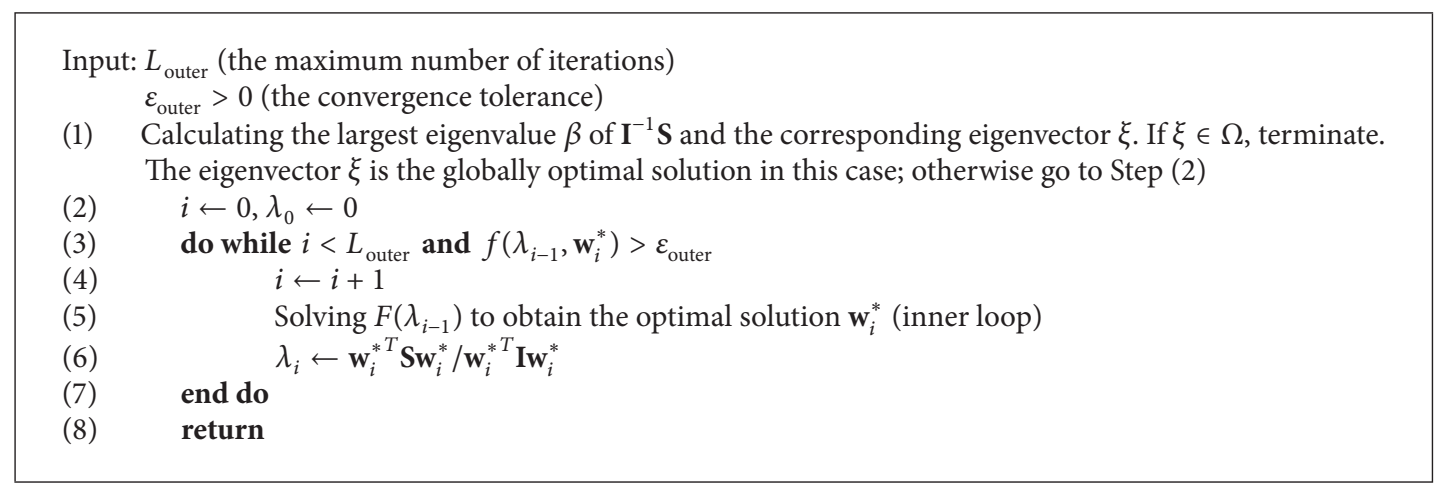

Algorithm 1: Iterative algorithm for SIR maximization.

In the equation, $\mathbf{M} \succ 0$ indicates that $\mathbf{M}$ is PSD. It is clear that except for the nonconvex rank-one constraint $\operatorname{rank}(\mathbf{M})=1$ in $C 5$, the remaining problem is convex. Finally, by dropping the nonconvex constraint, we can obtain a relaxed semidefinite programming (SDP) problem, which can be efficiently solved using the general purpose CVX package [22].

\section{Numerical Results}

In this section, the SIR performance of the proposed pulseshaping algorithm is evaluated. The set of prototype pulses such as Hermite, IOTA, SRRC, and OFDP will serve as a basis for our comparison. The CP-OFDM with rectangular pulse is also included for comparison. The experiments are established through a $20 \mathrm{MHz}$ channel for OFDM/OQAM systems where $M=256$ subcarriers and 4-QAM are employed. The one-tap ZF equalizer is used at the output of the demodulator to compensate the phase rotation caused by CFO and TO. The WSSUS channel with exponential power delay profile and U-shape Doppler spectrum is adopted. The rectangular time-frequency lattice is set to adapt to the channel property, that is, $\tau_{0} / F_{0}=\tau_{\max } / f_{d}$. Therefore, the transmission pattern of the system is fixed. In addition, $\Delta f$ and $\Delta t$ are normalized to $F_{0}$ and $\tau_{0}$, respectively.

The time and frequency responses of the optimal pulse are shown in Figure 2 when the channel spread factor is fixed to 0.01 and both of the normalized CFO and TO are fixed to 0.05 . In the figure, the curves of rectangular pulse and OFDP are included for comparison. It is clear that the OFDP yields a better result in frequency domain. On the other hand, the optimal pulse has a better SIR performance in the presence of desynchronization over DD channels which will be confirmed in the following simulations.

It is obvious that the SIR performance will be improved while increasing the number $L$ of Hermite functions. Nevertheless, the improvement decreases with $L$. Meanwhile, increasing $L$ will induce computational complexity and even cause instability in the optimization process. Thus, we choose $L=5$ as a compromise.

Figure 3 shows the SIR performance of the considered prototype pulses as a function of the channel spread factor $\theta$ when the synchronization is perfect (i.e., $\Delta f=\Delta t=$ 0 ). In the simulation, the optimization is redone for each set of $\theta$. From the figure, it can be seen that the optimal design significantly outperforms other prototype pulses when $\theta \geq 0.006$. However, the SIR is about only $27 \mathrm{~dB}$ when $\theta=0$ due to the fact that the constraints on the nulls of the ambiguity function of the proposed pulse are abandoned. The Hermite pulse and IOTA pulse lead to approximately the same performance, so IOTA is not included in the following comparisons.

The SIR curves as a function of the normalized CFO are presented in Figures 4 and 5 for the case where timing is perfect. In the scenario, we set the channel spread factor $\theta=0.003$ in Figure 4 and $\theta=0.018$ in Figure 5. The optimal pulse is uniquely determined by a fixed CFO $f_{e}$ which is set to 0.05 in the simulations. From the figures, an improvement of the robustness against the CFO can be addressed by using the optimal pulse. By varying $f_{e}$, we can get a series of optimal pulses and the corresponding maximum SIR. Then, the SIR upper bound can be obtained which shows the improvement margin for a given CFO. It can also be concluded that when the optimal pulse is employed, more SIR performance gain can be obtained for larger CFO and channel spread factor.

Figures 6 and 7 show the SIR results for the case when $\Delta f=0$. The results are given as a function of the normalized TO for a fixed channel spread factor $\theta=0.003$ in Figure 6 and $\theta=0.018$ in Figure 7 . In the simulations, we set the optimized point $t_{e}=0.03$. As is shown in the figures, except for the rectangular pulse in OFDM, the optimal pulse has the highest SIR at the optimized point. CP-OFDM perfectly suppresses the interference caused by TO within cyclic prefix but performs poorly over DD channels. By varying $t_{e}$, we can also get a SIR upper bound. Similar results can be concluded that more SIR performance gain can be obtained for larger TO and channel spread factor.

We also note that, in Figures 4 and 6, where the small value of channel spread factor is used, the optimal pulse performs slightly inferior to other pulses in the small CFO and TO regions. However, the SIR level is still higher than $20 \mathrm{~dB}$. 


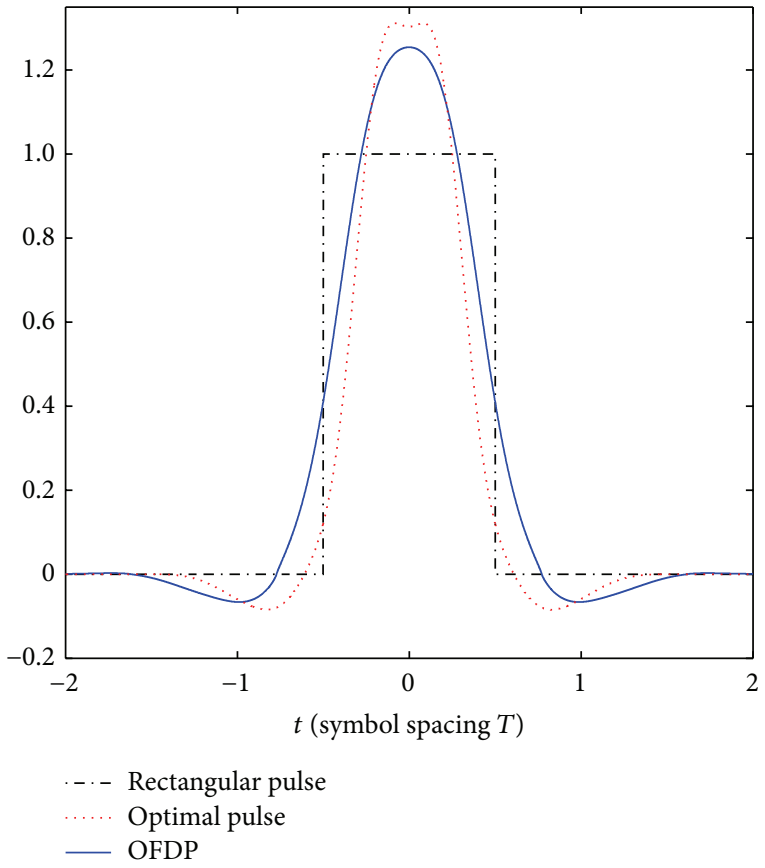

(a) Time domain pulses

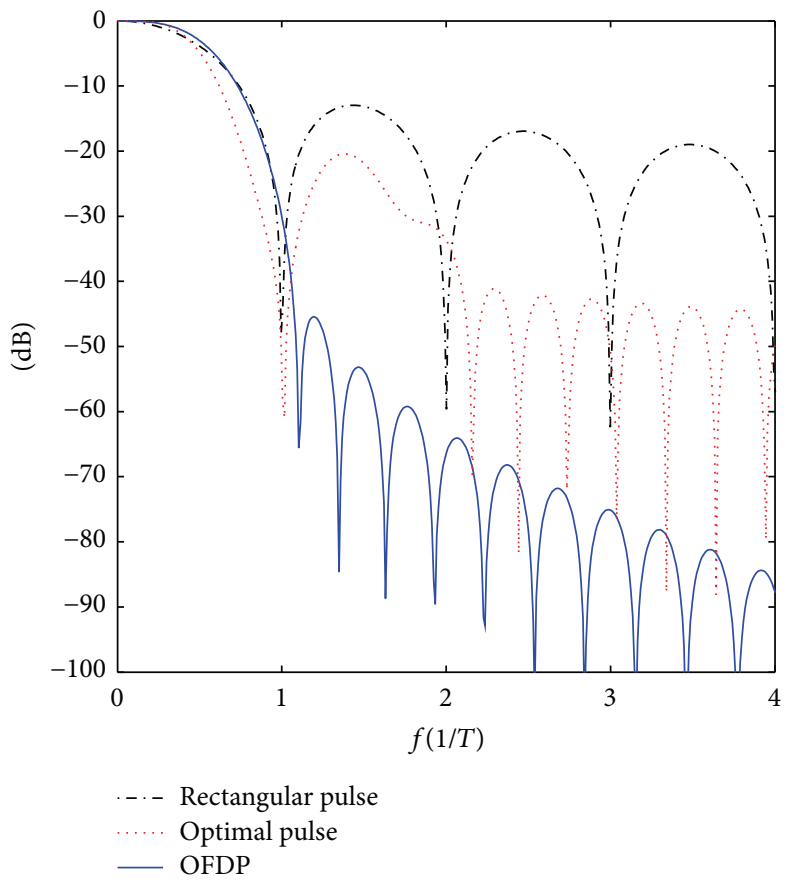

(b) Frequency domain pulses

FIgURE 2: Pulse shapes in time and frequency domain.

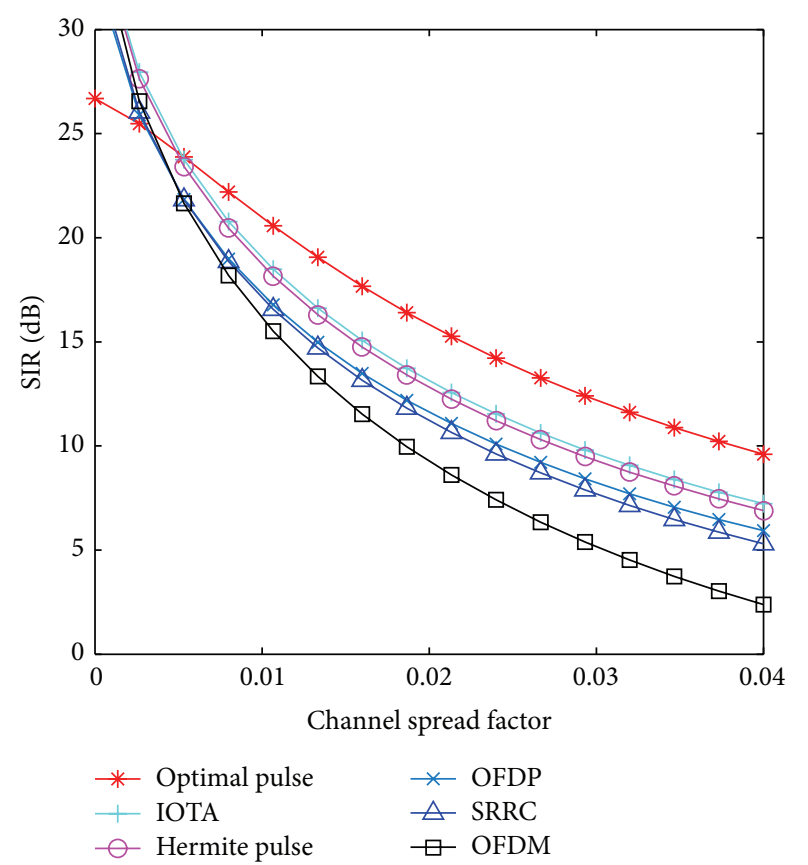

FIGURE 3: SIR as a function of channel spread factor with perfect synchronization.

Figure 8 gives the SIR comparison where the same increment step for $\mathrm{CFO}$ and $\mathrm{TO}$ is set. we set $\theta=0.01$ and the optimized point $t_{e}=f_{e}=0.05$. The result

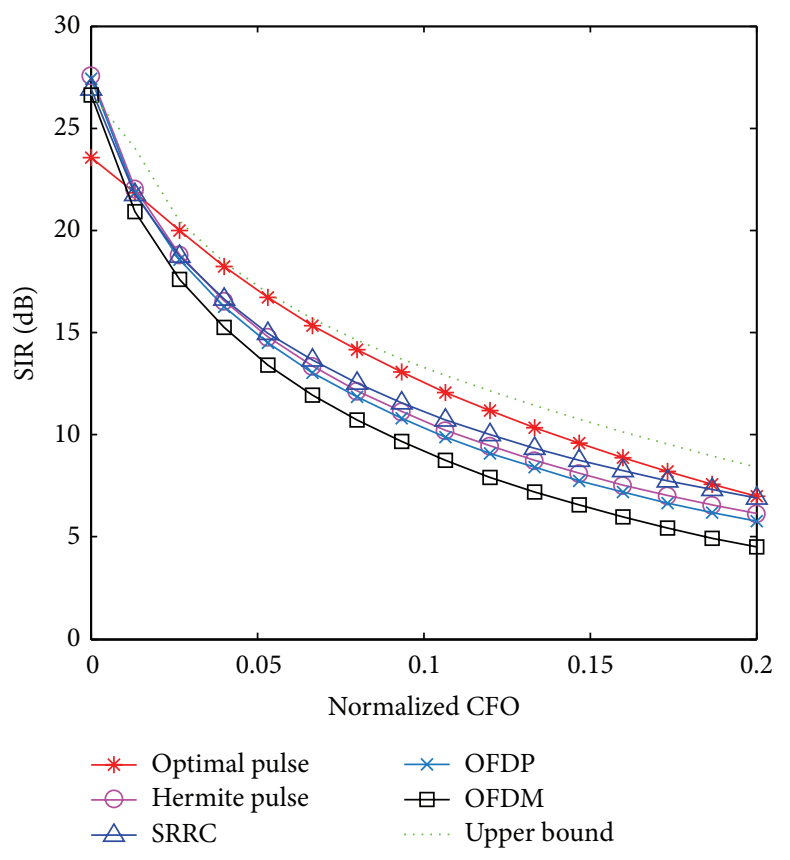

FIGURE 4: SIR as a function of normalized CFO for $\Delta t=0$ over a DD channel with $\theta=0.003$.

confirms once again the efficiency of our method to acquire a prototype pulse robust to insufficient synchronization over DD channels. 


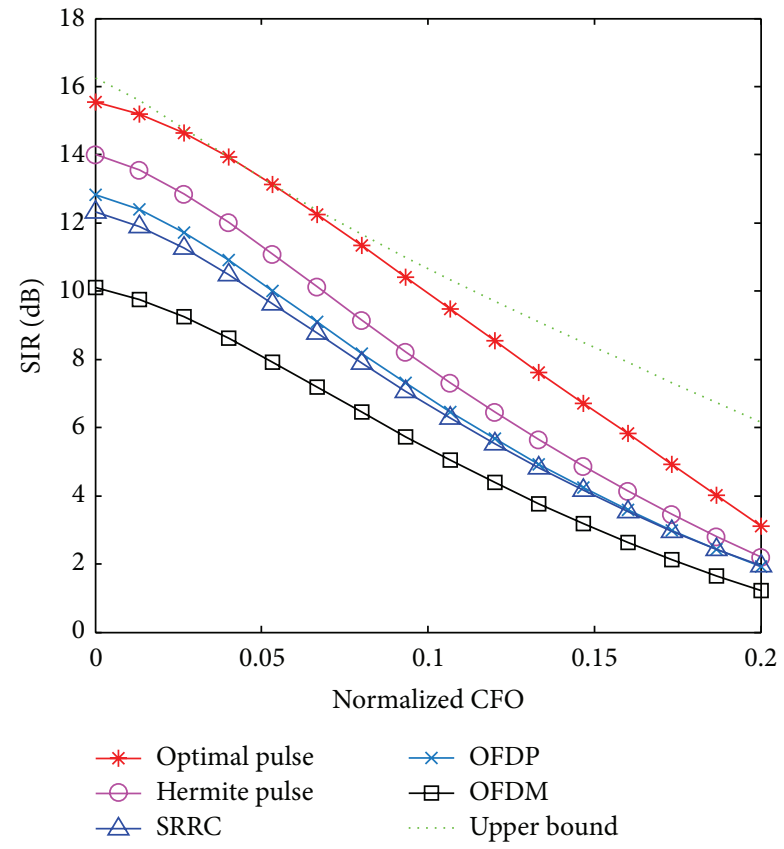

FIGURE 5: SIR as a function of normalized CFO for $\Delta t=0$ over a DD channel with $\theta=0.018$.

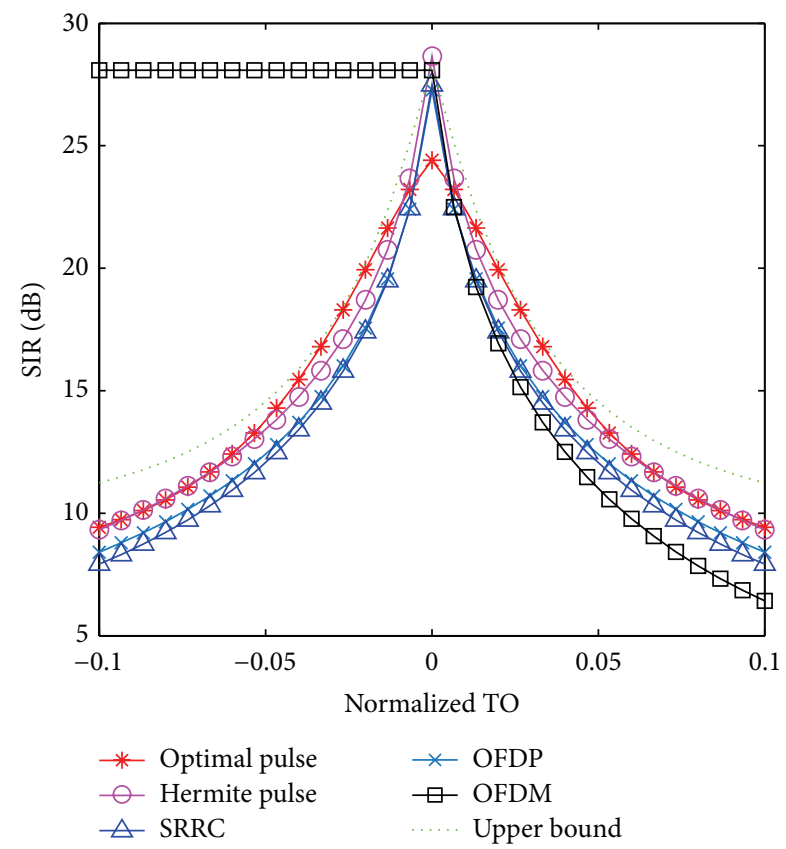

FIGURE 6: SIR as a function of normalized TO for $\Delta f=0$ over a DD channel with $\theta=0.003$.

\section{Conclusion}

In this paper, we have proposed a procedure for designing prototype pulses for OFDM/OQAM systems that are optimal with respect to SIR performance over doubly dispersive channels with insufficient synchronization. Based on SIR

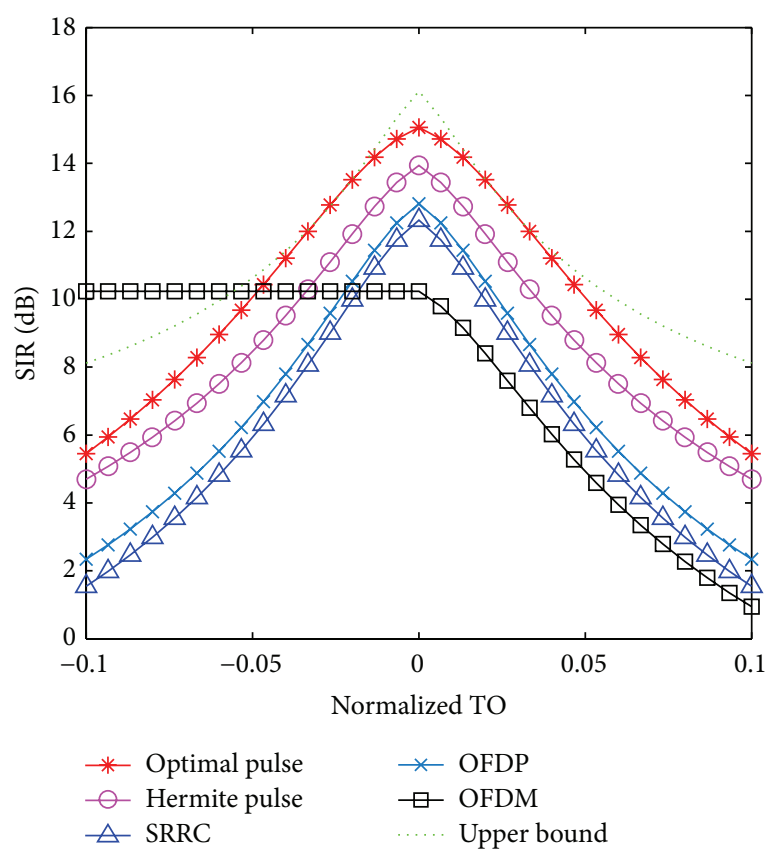

FIGURE 7: SIR as a function of normalized TO for $\Delta f=0$ over a DD channel with $\theta=0.018$.

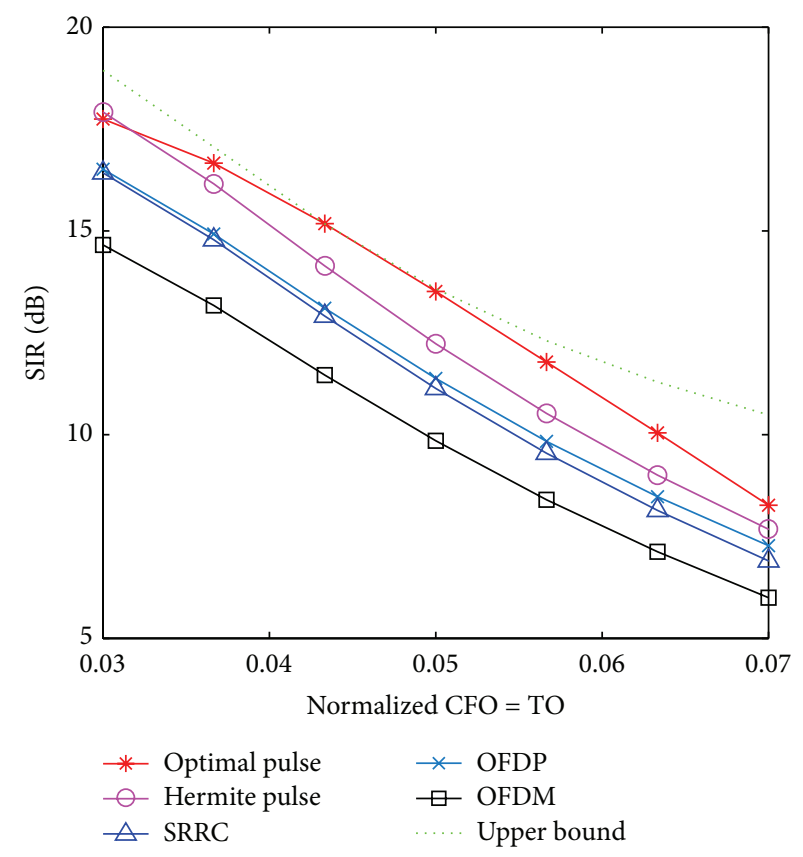

FIgURE 8: SIR as a function of the equivalent $\mathrm{CFO}$ and TO with $\theta=$ 0.01 .

maximization criterion, the pulse design procedure has been formulated as a nonconvex fractional programming problem which was efficiently solved by employing an iterative algorithm. Numerical simulations demonstrated that the optimal pulse provides a significant performance gainover traditional 
pulses. The advantage is especially prominent in the case of seriously dispersive channels with large carrier frequency and timing offset.

\section{Appendices}

\section{A. Derivation of (12)}

Consider

$$
\begin{aligned}
& \Theta_{m_{0}, k_{0} ; m, k}^{\Delta t, \Delta f}=\left\langle e^{j 2 \pi \Delta f t} H\left(g_{m, k}(t)\right), g_{m_{0}, k_{0}}(t-\Delta t)\right\rangle=\int_{t} e^{j 2 \pi \Delta f t} \int_{\tau} \int_{v} H(\tau, v) g_{m, k}(t-\tau) e^{j 2 \pi v t} g_{m_{0}, k_{0}}^{*}(t-\Delta t) d \tau d v d t \\
& =e^{j\left(\phi_{m k}-\phi_{m_{0} k_{0}}\right)} e^{j 2 \pi m_{0} F_{0} \Delta t} \int_{\tau} \int_{v} \int_{t} H(\tau, v) e^{j 2 \pi m F_{0}(t-\tau)} e^{j 2 \pi\left(\Delta f t-m_{0} F_{0} t+v t\right)} g^{*}\left(t-\Delta t-k_{0} \tau_{0}\right) g\left(t-\tau-k \tau_{0}\right) d t d \tau d v \\
& =e^{j\left(\phi_{m k}-\phi_{m_{0} k_{0}}\right)} e^{j 2 \pi\left[\left(\left(m-m_{0}\right) F_{0}+\Delta f\right)\left(\Delta t+k_{0} \tau_{0}\right)+m_{0} F_{0} \Delta t\right]} \int_{\tau} \int_{v} H(\tau, v) \\
& \cdot e^{j 2 \pi\left[v\left(\Delta t+k_{0} \tau_{0}\right)-m F_{0} \tau\right]} A_{g}^{*}\left(\left(k-k_{0}\right) \tau_{0}+\tau-\Delta t,\left(m-m_{0}\right) F_{0}+v+\Delta f\right) d \tau d v .
\end{aligned}
$$

By taking into account the ZF equalizer coefficient $H_{m_{0} k_{0}}^{\prime}$, we can obtain

$$
\begin{aligned}
& \frac{\Theta_{m_{0}, k_{0} ; m, k}^{\Delta t, \Delta f}}{H_{m_{0} k_{0}}^{\prime}} \\
& \quad=e^{j\left(\phi_{m k}-\phi_{m_{0} k_{0}}\right)} e^{j 2 \pi\left(m-m_{0}\right) F_{0}\left(\Delta t+k_{0} \tau_{0}\right)} \int_{\tau} \int_{v} H(\tau, v) \\
& \cdot e^{j 2 \pi\left[v\left(\Delta t+k_{0} \tau_{0}\right)-m F_{0} \tau\right]} A_{g}^{*}\left(\left(k-k_{0}\right) \tau_{0}+\tau\right. \\
& \left.-\Delta t,\left(m-m_{0}\right) F_{0}+v+\Delta f\right) d \tau d v \\
& =e^{j\left(\phi_{m k}-\phi_{m_{0} k_{0}}\right)} \Xi_{m_{0}, k_{0} ; m, k}^{\Delta t, \Delta f}
\end{aligned}
$$

We assume that the spreading function $H(\tau, v)$ is circularsymmetric (real and imaginary sections are uncorrelated and have the same variance). Then,

$$
\begin{aligned}
& E\left\{\left|\Re\left\{\frac{\Theta_{m_{0}, k_{0} ; m, k}^{\Delta t, \Delta f}}{H_{m_{0} k_{0}}^{\prime}}\right\}\right|^{2}\right\} \\
& =E\left\{\left|\mathfrak{R}\left\{e^{j\left(\phi_{m k}-\phi_{m_{0} k_{0}}\right)} \Xi_{m_{0}, k_{0} ; m, k}^{\Delta t, \Delta f}\right\}\right|^{2}\right\} \\
& =\frac{1}{2} E\left\{\left|\Xi_{m_{0}, k_{0} ; m, k}^{\Delta t, \Delta f}\right|^{2}\right\} .
\end{aligned}
$$

Considering the statistically independent property of symbols $a_{m, k}$ and the WSSUS assumption, the total energy of the equalized symbol can be calculated as

$$
\begin{aligned}
& E_{r}\left(\tau_{\max }, f_{d}, \Delta t, \Delta f\right) \\
& =E\left\{\left|\sum_{k \in \mathbb{Z}} \sum_{m=0}^{M-1} a_{m, k} \Re\left\{\frac{\Theta_{m_{0}, k_{0} ; m, k}^{\Delta t, \Delta f}}{H_{m_{0} k_{0}}^{\prime}}\right\}\right|^{2}\right\} \\
& =\frac{1}{2} E\left\{\left|\sum_{k \in \mathbb{Z}} \sum_{m=0}^{M-1} a_{m, k} \Xi_{m_{0}, k_{0} ; m, k}^{\Delta t, \Delta f}\right|^{2}\right\}
\end{aligned}
$$

$$
\begin{aligned}
& =\frac{\sigma_{a}^{2}}{2} \sum_{m} \sum_{k} \int_{\tau_{1}} \int_{v_{1}} \int_{\tau_{2}} \int_{v_{2}} E\left\{H^{*}\left(\tau_{1}, v_{1}\right) H\left(\tau_{2}, v_{2}\right)\right\} \\
& \cdot e^{-j 2 \pi m F_{0}\left(\tau_{2}-\tau_{1}\right)} e^{j 2 \pi\left(\Delta t+k_{0} \tau_{0}\right)\left(v_{2}-v_{1}\right)} \\
& \cdot A_{g}\left(\left(k-k_{0}\right) \tau_{0}+\tau_{1}-\Delta t,\left(m-m_{0}\right) F_{0}+v_{1}+\Delta f\right) \\
& \cdot A_{g}^{*}\left(\left(k-k_{0}\right) \tau_{0}+\tau_{2}-\Delta t\right. \\
& \left.\left(m-m_{0}\right) F_{0}+v_{2}+\Delta f\right) d \tau_{1} d v_{1} d \tau_{2} d v_{2} \\
& =\frac{1}{2} \sigma_{a}^{2} \sum_{k \in \mathbb{Z}} \sum_{m=0}^{M-1} \int_{\tau} \int_{v} S_{H}(\tau, v) \cdot \mid A_{g}\left(\left(k-k_{0}\right) \tau_{0}+\tau-\Delta t,\right. \\
& \left.\left(m-m_{0}\right) F_{0}+v+\Delta f\right)\left.\right|^{2} d \tau d v .
\end{aligned}
$$

\section{B. Constraints Caused by the Operation of Matrix Kronecker Product}

While referring to the expression of $\mathbf{w}$, we deduce that

(1) $w_{(i+1) L+j}=w_{j L+i+1}, 0 \leq i \leq L-2,0 \leq j \leq i$;

(2) $\left|w_{i L+j+1}\right|^{2}=w_{i L+i} w_{(j+1) L+j+1}, 0 \leq i \leq L-2, i \leq j \leq$ $L-2$;

(3) $w_{i L+i}=\left|\alpha_{2 i}\right|^{2} \geq 0,0 \leq i \leq L-1$.

From (1), the equality constraint can be summarized by $\mathbf{C w}=0$, where $\mathbf{C}$ is a $L^{2} \times L^{2}$ matrix defined by $\mathbf{C}=$

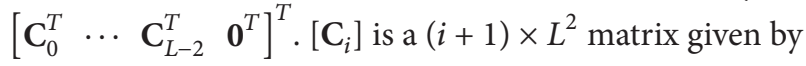

$$
\left[\mathbf{C}_{i}\right]_{m n}= \begin{cases}1 & n=(i+1) L+m \\ -1 & n=m L+i+1 \\ 0 & \text { otherwise }\end{cases}
$$


From (2), we can deduce the kind of equality constraint: $\mathbf{w}^{T} \mathbf{D}_{i j} \mathbf{w}=0$, where $\mathbf{D}_{i j}$ is a $L^{2} \times L^{2}$ matrix defined by $\mathbf{D}_{i j}=$ $\left[\begin{array}{lll}\mathbf{0}_{i L \times L^{2}}^{T} & \mathbf{E}_{i j}{ }^{T} & \mathbf{0}_{(L-(i+1)) L \times L^{2}}^{T}\end{array}\right]^{T} . \mathbf{E}_{i j}$ is a $L \times L^{2}$ matrix given by

$$
\left[\mathbf{E}_{i j}\right]_{m n}= \begin{cases}1 & m=j+1, n=i L+j+1 \\ -0.5 & m=i, n=(j+1) L+j+1 \\ -0.5 & m=(j+1) L+j+1, n=i \\ 0 & \text { otherwise. }\end{cases}
$$

From (3), we can deduce the inequality constraint: $\mathbf{G w} \geq$ 0 , where $\mathbf{G}$ is a $L^{2} \times L^{2}$ matrix defined by

$$
[\mathbf{G}]_{m n}= \begin{cases}1 & n=m(L+1), 0 \leq m \leq L-1 \\ 0 & \text { otherwise. }\end{cases}
$$

\section{Conflict of Interests}

The authors declare that there is no conflict of interests regarding the publication of this paper.

\section{Acknowledgment}

This work was supported by National Natural Science Fund of China under Grant no. 60971100.

\section{References}

[1] W. Kozek and A. F. Molisch, "Nonorthogonal pulseshapes for multicarrier communications in doubly dispersive channels," IEEE Journal on Selected Areas in Communications, vol. 16, no. 8, pp. 1579-1589, 1998.

[2] D. Schafhuber, G. Matz, and F. Hlawatsch, "Pulse-shaping OFDM/BFDM systems for time-varying channels: ISI/ICI analysis, optimal pulse design, and efficient implementation," in Proceedings of the 13th IEEE International Symposium on Personal, Indoor and Mobile Radio Communications (PIMRC '02), vol. 3, pp. 1012-1016, IEEE, Lisbon, Portugal, September 2002.

[3] K. Liu, T. Kadous, and A. M. Sayeed, "Orthogonal time-frequency signaling over doubly dispersive channels," IEEE Transactions on Information Theory, vol. 50, no. 11, pp. 2583-2603, 2004.

[4] T. Strohmer and S. Beaver, "Optimal OFDM design for timefrequency dispersive channels," IEEE Transactions on Communications, vol. 51, no. 7, pp. 1111-1122, 2003.

[5] B. Farhang-Boroujeny, "OFDM versus filter bank multicarrier," IEEE Signal Processing Magazine, vol. 28, no. 3, pp. 92-112, 2011.

[6] J. F. Du and S. Signell, "Classic OFDM systems and pulseshaping OFDM/OQAM systems," Tech. Rep., KTH-Royal Institute of Technology, 2007.

[7] H. Saeedi-Sourck, Y. Wu, J. W. M. Bergmans, S. Sadri, and B. Farhang-Boroujeny, "Sensitivity analysis of offset QAM multicarrier systems to residual carrier frequency and timing offsets," Signal Processing, vol. 91, no. 7, pp. 1604-1612, 2011.

[8] H. Lin, M. Gharba, and P. Siohan, "Impact of time and carrier frequency offsets on the FBMC/OQAM modulation scheme," Signal Processing, vol. 102, pp. 151-162, 2014.
[9] P. Jung and G. Wunder, "The WSSUS pulse design problem in multicarrier transmission," IEEE Transactions on Communications, vol. 55, no. 10, pp. 1918-1928, 2007.

[10] R. Ayadi, I. Kammoun, and M. Siala, "Optimization of the pulse shape of OFDM systems using the arrow-hurwicz algorithm," in Proceedings of the 4th IEEE International Symposium on Wireless Communication Systems (ISWCS '07), pp. 91-95, October 2007.

[11] P. Amini, R.-R. Chen, and B. Farhang-Boroujeny, "Filterbank multicarrier communications for underwater acoustic channels," IEEE Journal of Oceanic Engineering, vol. 40, no. 1, pp. 115130, 2014.

[12] G. Lin, L. Lundheim, and N. Holte, "Optimal pulses robust to carrier frequency offset for OFDM/QAM systems," IEEE Communications Letters, vol. 12, no. 3, pp. 161-163, 2008.

[13] P. Bello, "Characterization of randomly time-variant linear channels," IEEE Transactions on Communications, vol. 11, no. 4, pp. 360-393, 1963.

[14] P. Siohan, C. Siclet, and N. Lacaille, "Analysis and design of OFDM/OQAM systems based on filterbank theory," IEEE Transactions on Signal Processing, vol. 50, no. 5, pp. 1170-1183, 2002.

[15] B. Le Floch, M. Alard, and C. Berrou, "Coded orthogonal frequency division multiplex," Proceedings of the IEEE, vol. 83, no. 6, pp. 982-996, 1995.

[16] R. Haas and J.-C. Belfiore, "A time-frequency well-localized pulse for multiple carrier transmission," Wireless Personal Communications, vol. 5, no. 1, pp. 1-18, 1997.

[17] A. Vahlin and N. Holte, "Optimal finite duration pulses for OFDM," IEEE Transactions on Communications, vol. 44, no. 1, pp. 10-14, 1996.

[18] S. Aldirmaz, A. Serbes, and L. Durak-Ata, "Spectrally efficient OFDMA lattice structure via toroidal waveforms on the timefrequency plane," EURASIP Journal on Advances in Signal Processing, vol. 2010, Article ID 684097, 8 pages, 2010.

[19] C. H. Wilcox, "The synthesis problem for radar ambiguity functions," in Radar and Sonar I, pp. 252-253, Springer, New York, NY, USA, 1991.

[20] W. Dinkelbach, "On nonlinear fractional programming," Management Science, vol. 13, pp. 492-498, 1967.

[21] Z.-Q. Luo, W.-K. Ma, A. So, Y. Ye, and S. Zhang, "Semidefinite relaxation of quadratic optimization problems," IEEE Signal Processing Magazine, vol. 27, no. 3, pp. 20-34, 2010.

[22] M. Grant, S. Boyd, and Y. Ye, CVX: MATLAB Software for Disciplined Convex Programming, 2009, http://www.stanford.edu/ boyd/cvx. 


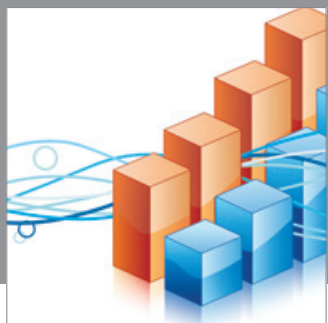

Advances in

Operations Research

mansans

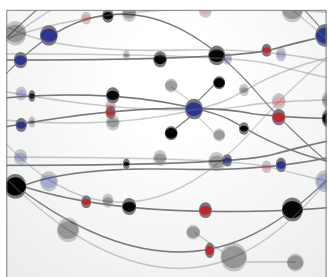

The Scientific World Journal
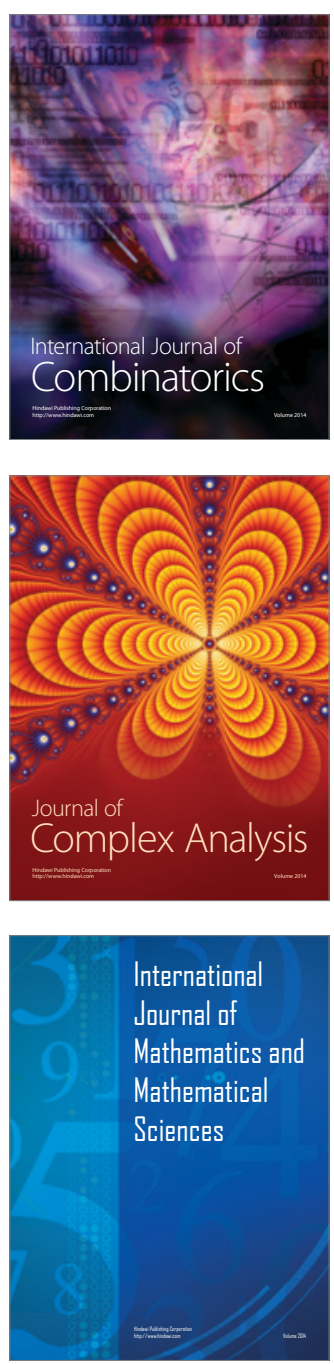
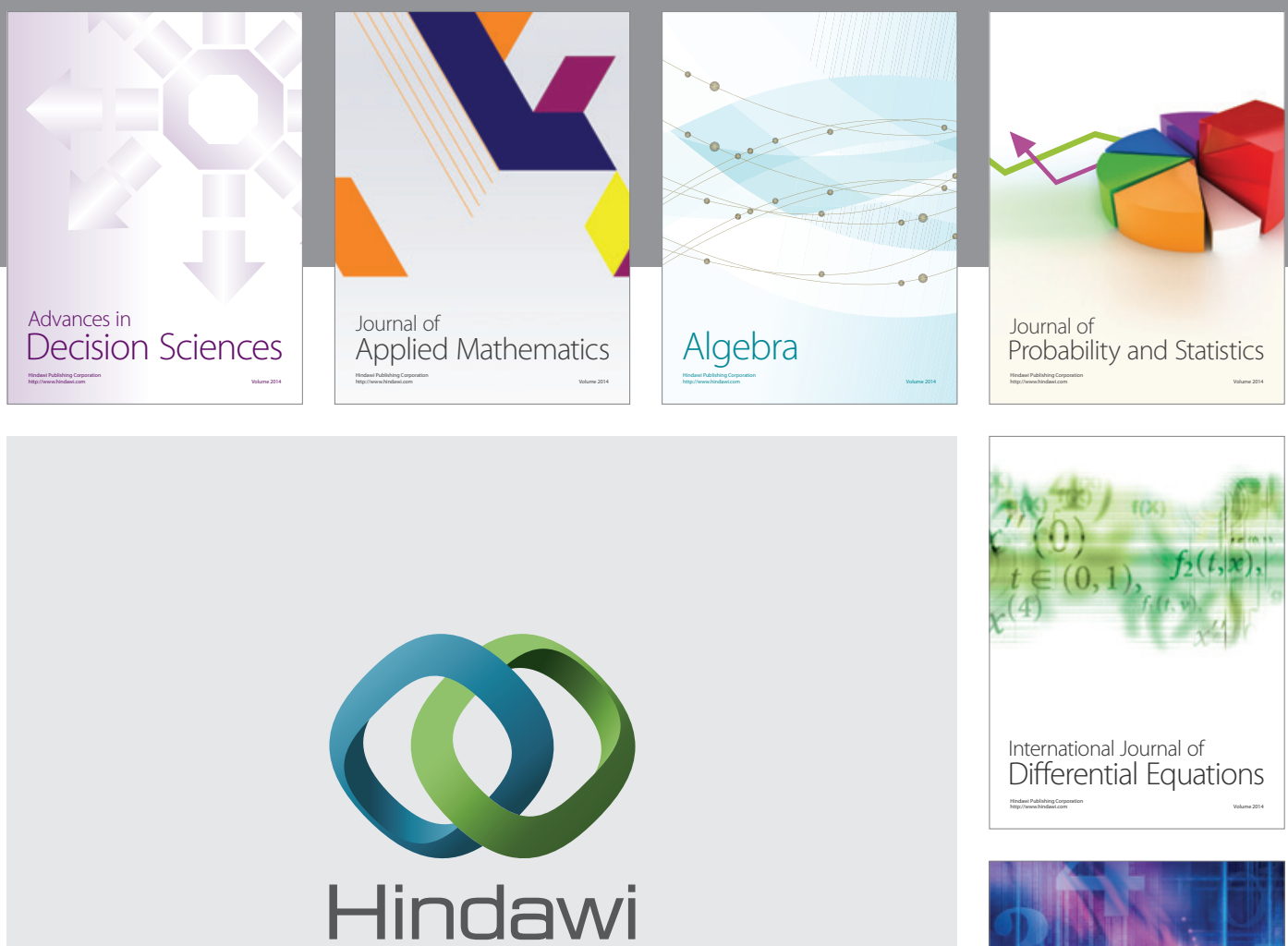

Submit your manuscripts at http://www.hindawi.com
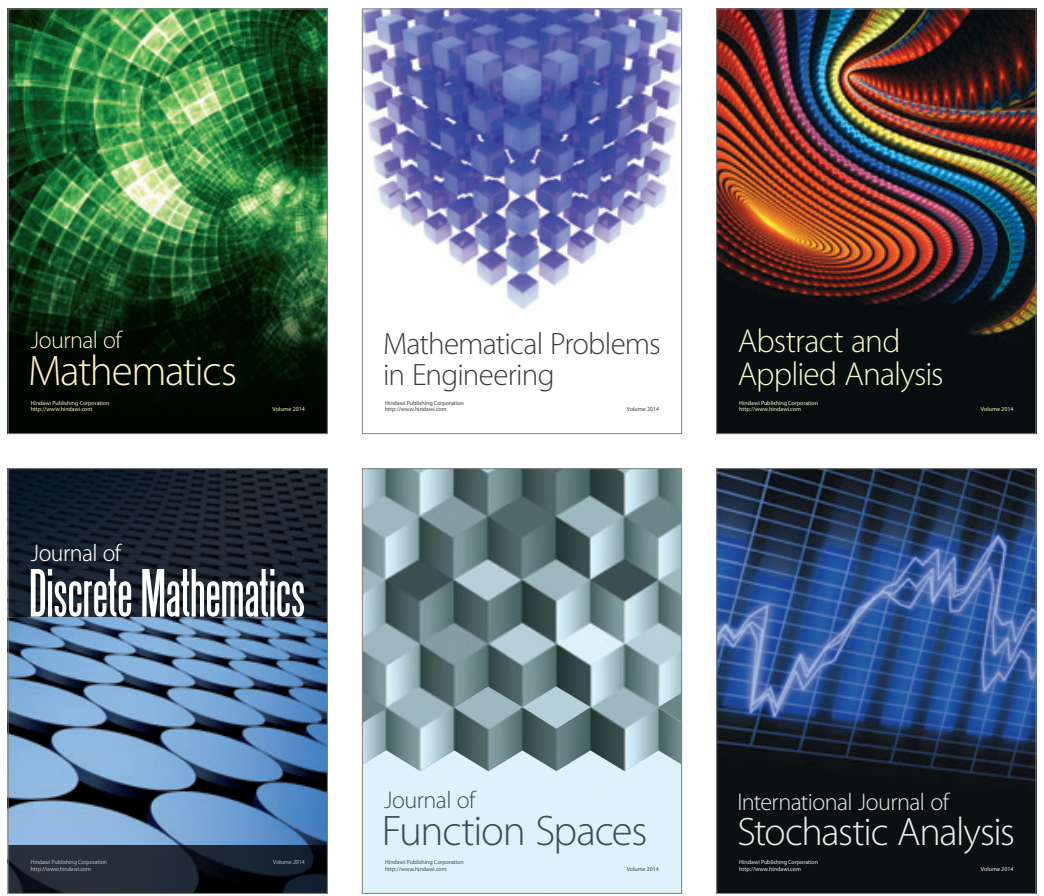

Journal of

Function Spaces

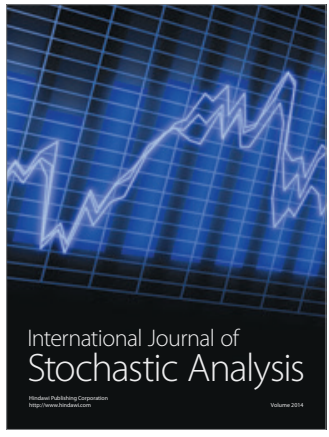

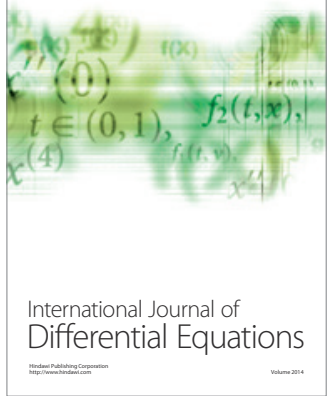
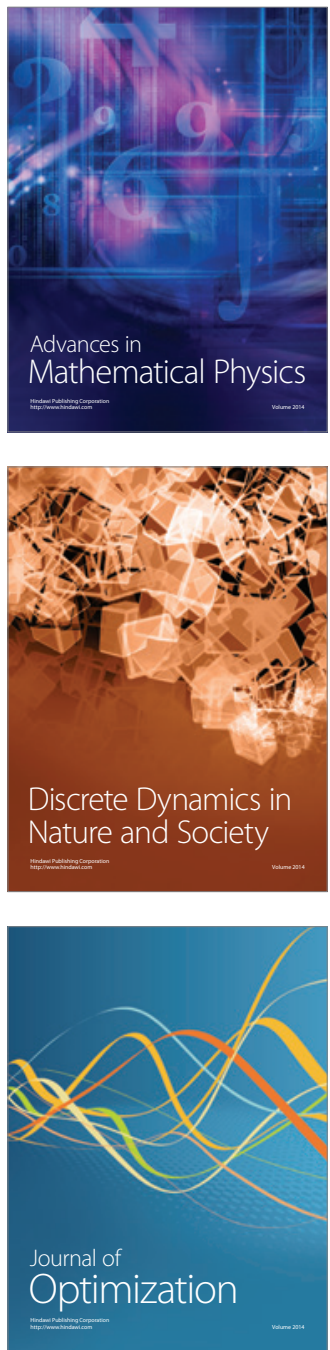